Texture of Crystalline Solids, 1981, Vol. 4, pp. 129-141

0390-7951/81/0403-0129\$06.50/0

(c) 1981 Gordon and Breach, Science Publishers, Inc.

Printed in the United States of America

\title{
TEXTURE AND MAGNETIC PROPERTIES
}

\author{
P. R. MORRIS and J. W. FLOWERS \\ Research and Technology, Armco Inc. \\ Middletown, Ohio 45043 U.S.A.
}

(Received November 18, 1980)

\begin{abstract}
Expressions are derived for the dependence of the magnetic properties of cubic materials on texture. The theoretical development generally parallels that of Bunge, but employs Roe's formalism. The expressions, together with limited experimental data, enable one to express magnetic property variations in the plane of the sheet, to obtain property values for comparable texture-free specimens, and to separate magnetic properties into texture-dependent and texture-independent components. Core loss, magnetic permeability and torque for specimens of "nonoriented" nominal 3\% silicon-iron are fitted to simple fourth-order equations.
\end{abstract}

\section{INTRODUCTION}

An equation giving the magnetization energy of cubic polycrystals as a function of direction in the plane of a sheet from the magneto-crystalline anisotropy coefficients and the coefficients of a spherical surface harmonic expansion of a pole figure was first derived by Bunge. I Development of methods by Bunge ${ }^{2}$ and Roe $^{3}, 4$ in which generalized spherical harmonic expansions of the crystallite orientation distribution (COD) were obtained from spherical surface harmonic expansions of the pole figure made it possible to express the orientation dependence of the magnetization energy of a polycrystal in terms of the coefficients of the series development of the COD. This was done for orthorhombic (sheet) sample symmetry and cubic crystal symmetry by Bunge. ${ }^{5}$ Hutchinson and Swift ${ }^{6}$ used Williams ${ }^{7}$ numerical technique to obtain polycrystal averages for power loss and torque as a function of direction in the plane of the sheet. Szpunar and Ojanen ${ }^{8}$ used the method of Bunge ${ }^{1}$ to calculate torque curves from individual (110), (200), and (211) pole figures. They found that results obtained with individual pole figures were in disagreement, and recommended that the coefficients of the series expansions of the individual pole figures be recalculated from the coefficients of the COD. As Bunge has shown, 5 such calculations can be carried out directly with 
the coefficients of the COD, making recalculation of the pole figure coefficients unnecessary for this purpose.

In the present work we present a theoretical development which generaliy parallels that of Bunge, 5 but employs the formalism of Roe. ${ }^{3}, 4$ A principal advantage of these techniques results from their ability to separate texture-dependent and texture-independent contributions to such properties as magnetic permeability and core loss, as noted by Hutchinson and Swift. 6

\section{THEORETICAL DISCUSSION}

Many physical properties of a single crystal may be approximated by a polynomial series expansion in which each term of the series is invariant with respect to crystal symmetry operations. Certain properties of cubic crystals may be represented by

$$
F(\vec{h})=B_{0}+B_{4} \dot{\dot{k}}_{4}(\vec{h})+B_{6} \dot{\mathrm{k}}_{6}(\vec{h})+\ldots,
$$

Where $F(\vec{h})$ denotes the value of the property in the direction $\overrightarrow{\mathrm{h}}$ referred to crystal fixed coordinates, $\dot{\mathrm{k}}_{4}(\overrightarrow{\mathrm{h}}), \dot{\mathrm{k}}_{6}(\overrightarrow{\mathrm{h}}), \ldots$ are "cubic spherical functions" of order $4,6, \ldots$, and $B_{0}$, $\mathrm{B}_{4}, \mathrm{~B}_{6} \ldots$ are coefficients of the series expansion. The cubic spherical functions are a series of orthonormal functions having cubic crystal symmetry, i.e.,

$$
\int_{-1}^{l}(d \cos \theta) \int_{0}^{2 \pi} \dot{\dot{k}}_{\ell}(\vec{h}) \dot{k}_{m}(\vec{h}) d \phi=\delta_{\ell m},
$$

where $\delta_{\ell m}$ is the Kronecker delta,

$$
\delta_{\ell m}=\left\{\begin{array}{ll}
1 & \text { if } \ell=m \\
0 & \text { if } \ell \neq m
\end{array},\right.
$$

and $\phi$ and $\theta$ are longitude angle measured from $X$ toward $Y$ crystallographic axes and colatitude angle measured from the Z crystallographic axis, respectively. Specifically,

$$
\begin{aligned}
& \dot{\mathrm{k}}_{4}(\overrightarrow{\mathrm{h}})=\left(\frac{42}{\pi}\right)^{\frac{1}{2}} \frac{\overline{\mathrm{P}}_{4}^{0}(\cos \theta)}{12}+\left(\frac{5}{3 \pi}\right)^{\frac{1}{2}} \frac{\overline{\mathrm{P}}_{4}^{4}(\cos \theta) \cos 4 \phi}{2}, \\
& \dot{\mathrm{k}}_{6}(\overrightarrow{\mathrm{h}})=\frac{1}{4 \sqrt{\pi}} \overline{\mathrm{P}}_{6}^{0}(\cos \theta)-\frac{1}{2}\left(\frac{7}{2 \pi}\right)^{\frac{1}{2}} \overline{\mathrm{P}}_{6}^{4}(\cos \theta) \cos 4 \phi,
\end{aligned}
$$

where $\overline{\mathrm{P}}_{\ell}^{\mathrm{m}}(\cos \theta)$ are the normalized associated Legendre functions, $\overline{\mathrm{P}}_{4}^{0}(\cos \theta)=\frac{3 \sqrt{2}}{128}(9+20 \cos 2 \theta+35 \cos 4 \theta)$, $\overline{\mathrm{P}}_{4}^{4}(\cos \theta)=\frac{3 \sqrt{35}}{128}(3-4 \cos 2 \theta+\cos 4 \theta)$, $\bar{P}_{6}^{0}(\cos \theta)=\frac{\sqrt{26}}{1024}(50+105 \cos 2 \theta+126 \cos 4 \theta+231 \cos 6 \theta)$, 
and $\bar{P}_{6}^{4}(\cos \theta)=\frac{3 \sqrt{91}}{1024}(10+5 \cos 2 \theta-26 \cos 4 \theta+11 \cos 6 \theta)$.

The inverse pole figure for a direction lying in the plane of the sheet at an angle a from the rolling direction is given by

$$
\begin{aligned}
P(\overrightarrow{\mathrm{h}})= & \frac{1}{4 \pi}+24 \pi \sqrt{\frac{\pi}{42}}\left[\frac{3}{8} \mathrm{~W}_{400}-\frac{\sqrt{10}}{4} \mathrm{~W}_{420} \cos 2 \alpha\right. \\
& \left.+\frac{\sqrt{70}}{8} \mathrm{~W}_{440} \cos 4 \alpha\right] \dot{\mathrm{k}}_{4}(\overrightarrow{\mathrm{h}})+8 \pi \sqrt{\pi}\left[-\frac{5}{16} \mathrm{~W}_{600}\right. \\
& +\frac{\sqrt{105}}{16} \mathrm{~W}_{620} \cos 2 \alpha-\frac{3 \sqrt{14}}{16} \mathrm{~W}_{640} \cos 4 \alpha \\
& \left.+\frac{\sqrt{231}}{16} \mathrm{~W}_{660} \cos 6 \alpha\right] \dot{\mathrm{k}}_{6}(\overrightarrow{\mathrm{h}})+\ldots
\end{aligned}
$$

where $P(\vec{h})$ has an average value of $I /(4 \pi)$, and $W_{l m n}$ are the coefficients of the generalized spherical harmonic expansion of the crystalljte orientation distribution in Roe's notation. The value of $F(\vec{h})$, averaged over all crystal directions lying in the plane of the sheet at an angle $\alpha$ to the rolling direction is obtained by multiplying $F(\vec{h})$ from Equation (I) by $P(\vec{h})$ from Equation (6), and integrating over all crystal orientations $\vec{h}$ to obtain

$$
\begin{aligned}
\bar{F}(\alpha)= & B_{0}+3 \pi \sqrt{\frac{\pi}{42}}\left(3 W_{400}-2 \sqrt{10} W_{420} \cos 2 \alpha+\right. \\
& \left.+\sqrt{70} W_{440} \cos 4 \alpha\right) B_{4}+\frac{\pi \sqrt{\pi}}{2}\left(-5 W_{600}+\sqrt{105} W_{620} \cos 2 \alpha\right. \\
& \left.-3 \sqrt{14} W_{640} \cos 4 \alpha+\sqrt{23 I} W_{660} \cos 6 \alpha\right) B_{6}+\ldots .
\end{aligned}
$$

Equation (7) may be simplified to

$$
\bar{F}(\alpha)=B_{0}+B_{4} T_{4}(\alpha)+B_{6} T_{6}(\alpha)+\ldots .
$$

If measured values of $\bar{F}\left(\alpha_{i}\right)$ are available for $i=1,2, \ldots, n$ values of $\alpha_{i}$, a least squares technique may be used to determine $\mathrm{B}_{0}, \mathrm{~B}_{4}, \mathrm{~B}_{6}$.

$$
\sum_{i=1}^{n} \frac{\partial}{\partial B_{j}}\left[B_{0}+B_{4} T_{4}\left(\alpha_{i}\right)+B_{6} T_{6}\left(\alpha_{i}\right)-\bar{F}\left(\alpha_{i}\right)\right]^{2}=0,
$$

$j=0,4,6$, leading to the normal equations

$$
B_{0} n+B_{4} \sum_{i=1}^{n} T_{4}\left(\alpha_{i}\right)+B_{6} \sum_{i=1}^{n} T_{6}\left(\alpha_{i}\right)=\sum_{i=1}^{n} \bar{F}\left(\alpha_{i}\right),
$$


$B_{0} \sum_{i=1}^{n} T_{4}\left(\alpha_{i}\right)+B_{4} \sum_{i=1}^{n}\left[T_{4}\left(\alpha_{i}\right)\right]^{2}+B_{6} \sum_{i=1}^{n} T_{4}\left(\alpha_{i}\right) T_{6}\left(\alpha_{i}\right)=\sum_{i=1}^{n} \bar{F}\left(\alpha_{i}\right) T_{4}\left(\alpha_{i}\right)$

$B_{0} \sum_{i=1}^{n} T_{6}\left(\alpha_{i}\right)+B_{4} \sum_{i=1}^{n} T_{4}\left(\alpha_{i}\right) T_{6}\left(\alpha_{i}\right)+B_{6} \sum_{i=1}^{n}\left[T_{6}\left(\alpha_{i}\right)\right]^{2}=\sum_{i=1}^{n} \bar{F}\left(\alpha_{i}\right) T_{6}\left(\alpha_{i}\right)$

Equations (10) are of the form

$$
[\mathrm{X}][\mathrm{B}]=[\mathrm{Y}] \text {. }
$$

and have a solution of the form

$$
[\mathrm{B}]=[\mathrm{X}]^{-1}[\mathrm{Y}] \text {. }
$$

Once $\mathrm{B}_{0}, \mathrm{~B}_{4}, \mathrm{~B}_{6}$ have been determined according to Equation (12), the variance $S_{F}^{2}$ of the $\bar{F}\left(\alpha_{i}\right)$ may be estimated according to

$$
S_{F}^{2}=\left(\frac{1}{n-m}\right)_{i=1}^{n}\left[B_{0}+B_{4} T_{4}\left(\alpha_{i}\right)+B_{6} T_{6}\left(\alpha_{i}\right)-\bar{F}\left(\alpha_{i}\right)\right]^{2}
$$

where $\mathrm{n}$ is the number of measured values $\bar{F}\left(\alpha_{i}\right)$ and $\mathrm{m}$ is the number of coefficients $\mathrm{B}_{0}, \mathrm{~B}_{4}, \mathrm{~B}_{6}$ determined. The variance of the coefficients $\mathrm{B}_{0}, \mathrm{~B}_{4}, \mathrm{~B}_{6}$ may then be estimated accord-. ing to

$$
\begin{aligned}
& S_{B_{0}}^{2}=x_{1}^{-1} S_{F}^{2}, \\
& S_{B_{4}}^{2}=x_{2}^{-\frac{1}{2}} S_{F}^{2}, \text { and } \\
& S_{B_{6}}^{2}=x_{3}^{-\frac{1}{3}} S_{F}^{2},
\end{aligned}
$$

where $x_{i}^{-1}$ are the elements of the inverse $[x]^{-1}$ of the matrix $[\mathrm{X}]$. The standard deviations of the $\mathrm{B}_{j}$ may then be estimated by taking the square roots of Equations (14). If the standard deviation of the $B_{j}$ is equal to or greater than the $B_{j}$, the experimental data do not justify the solution, and a lower order series approximation should be used. Once the $\mathrm{B}_{j}$ have been obtained, $F(\alpha)$ may be calculated according to Equation (7). The orientation independent contribution to the measured property is given by $\mathrm{B}_{0}$. The average value of the property in the plane of the sheet is given by

$$
\mathrm{B}_{0}+9 \pi \sqrt{\frac{\pi}{42}} \mathrm{~W}_{400} \mathrm{~B}_{4}-\frac{5 \pi \sqrt{\pi}}{2} \mathrm{~W}_{6} 00 \mathrm{~B}_{6} \text {. }
$$

Thus, $9 \pi \sqrt{\frac{\pi}{42}} \mathrm{~W}_{4} 00 \mathrm{~B}_{4}-\frac{5 \pi \sqrt{\pi}}{2} \mathrm{~W}_{600} \mathrm{~B}_{6}$ represents the net gain (or loss) of the average property in the plane of the sheet due to the effect of preferred orientation. Once $B_{0}, B_{4}, B_{6}$ 
have been obtained, Equations (1), (4), and (5) may be used to calculate $F(\vec{h})$ for $\vec{h}$ corresponding to various crystallographic directions, if desired.

\section{Alternative Treatment}

An alternative representation has frequently been used to that of Equation (1),

$$
F(\vec{h})=A_{0}+A_{4} \phi_{4}(\vec{h})+A_{6} \phi_{6}(\vec{h})+\ldots .
$$

where $\phi_{4}(\vec{h})=l^{2} m^{2}+m^{2} n^{2}+n^{2} \ell^{2}, \phi_{6}(\vec{h})=l^{2} m^{2} n^{2}, l, m, n$ are direction cosines with respect to the $X, Y, Z$ cubic crystal axes, and higher order terms are formed by products of $\phi_{4}(\vec{h})$ and $\phi_{6}(\vec{h}), \phi_{8}(\vec{h})=\phi_{4}^{2}(\vec{h}), \phi_{10}(\vec{h})=\phi_{4}(\vec{h}) \phi_{6}(\vec{h}), \phi_{12}^{1}(\vec{h})=\phi_{4}^{3}(\vec{h})$, $\phi_{12}^{2}(\vec{h})=\phi_{6}^{2}(\vec{h})$, e.g. This representation has a disadvantage with respect to that of Equation (1), in that the $\dot{\hat{k}}_{i}(\vec{h})$ form a series of orthogonal functions [which simplifies integration of the product $F(\vec{h}) P(\vec{h})$ leading to Equation $(7)]$, while the $\phi_{i}(\vec{h})$ do not.

The $\phi_{i}(\dot{\vec{h}})$ may be expressed in terms of the $\dot{\mathrm{k}}_{i}(\overrightarrow{\mathrm{h}})$,

$$
\begin{aligned}
& \phi_{4}(\vec{h})=\frac{1}{5}\left[1+\frac{\dot{\mathrm{k}}_{4}(\overrightarrow{\mathrm{h}})}{\mathrm{n}_{4}}\right], \text { and } \\
& \phi_{6}(\overrightarrow{\mathrm{h}})=\frac{1}{105}-\frac{\dot{\mathrm{k}}_{4}(\overrightarrow{\mathrm{h}})}{55 \mathrm{n}_{4}}+\frac{\dot{\mathrm{k}}_{6}(\overrightarrow{\mathrm{h}})}{231 \mathrm{n}_{6}},
\end{aligned}
$$

where $n_{4}=-\left(\frac{21}{16 \pi}\right)^{\frac{1}{2}}$ and $n_{6}=\left(\frac{13}{128 \pi}\right)^{\frac{1}{2}}$. The coefficients of the expansions Equation (1) and Equation (15) are related by

$$
\begin{aligned}
& B_{0}=A_{0}+\frac{A_{4}}{5}+\frac{A_{6}}{105}, \\
& B_{4}=\frac{A_{4}}{5 n_{4}}-\frac{A_{6}}{55 n_{4}}, \text { and } \\
& B_{6}=\frac{A_{6}}{231 n_{6}},
\end{aligned}
$$

where truncation at order 6 has been assumed. By substitution of $\mathrm{B}_{0}, \mathrm{~B}_{4}, \mathrm{~B}_{6}$ from Equations (19) to (20) in Equation (8), we obtain

$$
\bar{F}(\alpha)=A_{0}+A_{4}\left[\frac{1}{5}+\frac{T_{4}(\alpha)}{5 n_{4}}\right]+A_{6}\left[\frac{1}{105}-\frac{T_{4}(\alpha)}{55 n_{4}}+\frac{T_{6}(\alpha)}{231 n_{6}}\right],
$$

where $T_{4}(\alpha), T_{6}(\alpha)$ are defined by Equations (7) and (8) . Equation (21) may be rewritten in the form

$$
\bar{F}(\alpha)=A_{0}+A_{4} \phi_{4}(\alpha)+A_{6} \phi_{6}(\alpha) \text {, where }
$$




$$
\begin{aligned}
& \phi_{4}(\alpha)=\frac{1}{5}+\frac{T_{4}(\alpha)}{5 n_{4}}, \text { and } \\
& \phi_{6}(\alpha)=\frac{1}{105}-\frac{T_{4}(\alpha)}{55 n_{4}}+\frac{T_{6}(\alpha)}{231 n_{6}} .
\end{aligned}
$$

The coefficients $A_{i}$ may be expressed in terms of the $B_{i}$ by

$$
\begin{aligned}
& A_{0}=B_{0}-n_{4} B_{4}-\frac{32}{5} n_{6} B_{6}, \\
& A_{4}=5 n_{4} B_{4}+21 n_{6} B_{6}, \text { and } \\
& A_{6}=231 n_{6} B_{6} .
\end{aligned}
$$

An expression for magnetic torque $\bar{M}(\alpha)$ in a homogeneous magnetic field in the direction $\alpha$ may be obtained by differentiating Equation (7) with respect to $\alpha$,

$$
\begin{aligned}
\bar{M}(\alpha)=- & \frac{d \bar{F}(\alpha)}{d \alpha}=12 \pi \sqrt{\frac{\pi}{42}}\left(-\sqrt{10} W_{420} \sin 2 \alpha+\sqrt{70} W_{440} \sin 4 \alpha\right) B_{4} \\
& +\pi \sqrt{\pi}\left(\sqrt{105} \mathrm{~W}_{620} \sin 2 \alpha-6 \sqrt{14} \mathrm{~W}_{640} \sin 4 \alpha\right. \\
& \left.+3 \sqrt{23 I} \mathrm{~W}_{66} 0 \sin 6 \alpha\right) \mathrm{B}_{6}+\ldots .
\end{aligned}
$$

\section{EXPERIMENTAL PROCEDURE}

The materials used for experimental work were specimens of nominal 3\% silicon-iron which are commercially designated as "nonoriented" electrical steels. Specimens A and C were cold-rolled to final thickness in a single-stage $81 \%$ cold reduction. Specimens $B$ and $D$ were cold-rolled to final thickness in two stages with cold reductions of $63 \%$ and $54 \%$, separated by an intermediate anneal. After cold rolling all specimens were annealed at $815^{\circ} \mathrm{C}$ to effect primary recrystallization and then annealed at $1040^{\circ} \mathrm{C}$ to allow some primary grain growth. After processing specimens $A$ and $B$ had grain sizes of about ASTM 1 while specimens $C$ and $D$ had grain sizes of about ASTM 5 .

Determinations of $\{110\},\{200\}$, and $\{112\}$ pole figures were made with an Enraf-Nonius automated X-ray diffraction unit on composite samples ${ }^{9}$ from fully-processed specimens. The pole figure data were used to calculate the COD to 16 th order by a method similar to one previously described. 10 Measurements of the magnetic permeability at $796 \mathrm{~A} / \mathrm{M}$ and the core loss at $1.5 \mathrm{~T}$ were made at $60 \mathrm{~Hz}$ on fully-processed samples from specimens $A$ and $B$ by conventional Epstein tests. The Epstein strips were given a stress-relief anneal at $815^{\circ} \mathrm{C}$ for $1 \mathrm{hr}$. prior to magnetic testing. Magnetic torque curves were determined for specimens $C$ and $D$ at Westinghouse Research Laboratories by a previously described method. ${ }^{1}$ 
RESULTS AND DISCUSSION

COD sections were obtained at constant $\phi$ values from $\phi=0^{\circ}$ to $\phi=90^{\circ}$ at $5^{\circ}$ intervals for fully-processed specimens. The $\phi=45^{\circ}$ sections for each specimen are given in Figure 1 . These sections contain the maximum COD values for
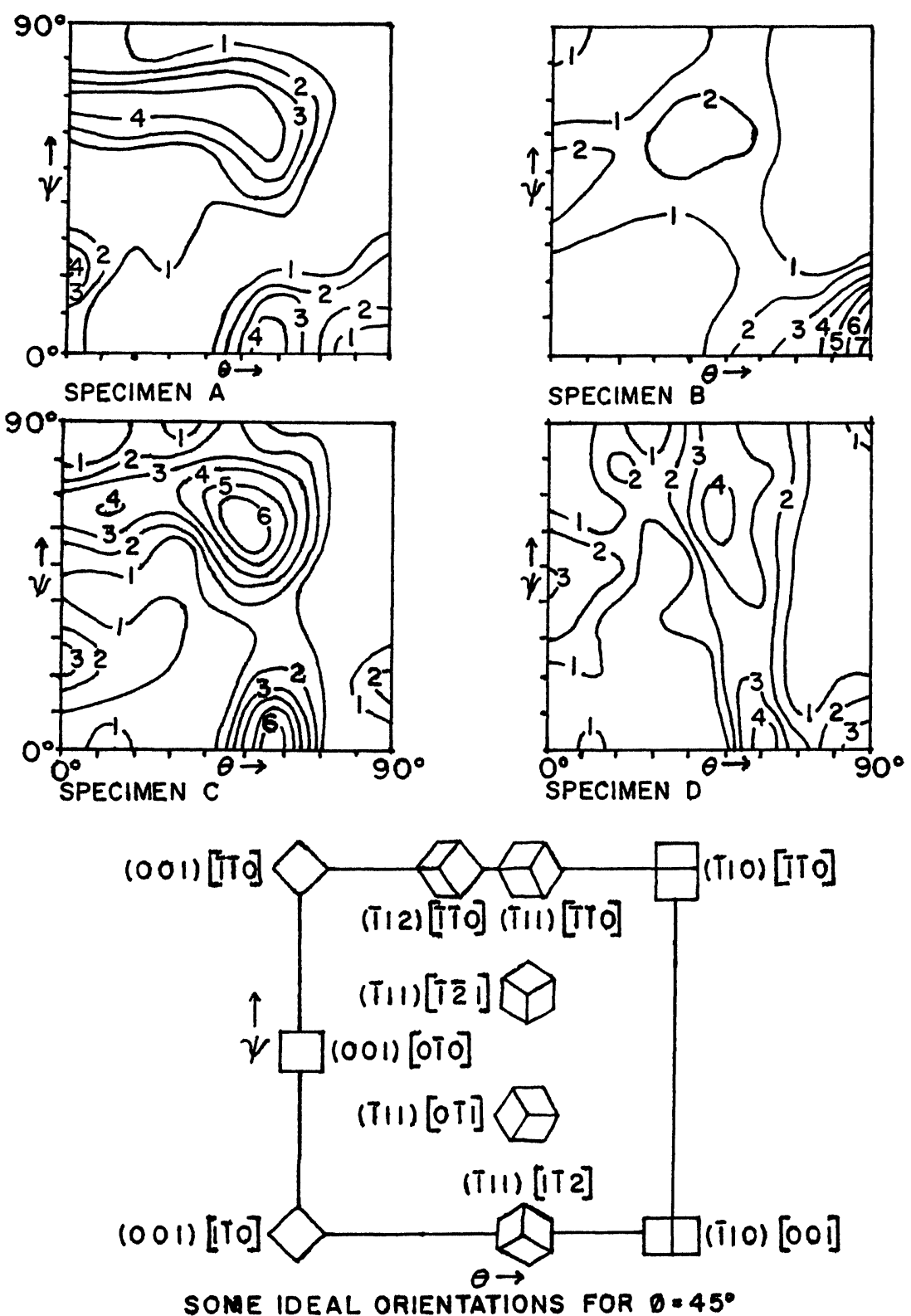

Figure 1. Crystallite orientation distribution function sections at $\phi=45^{\circ}$ for experimental specimens and schematic showing location of some ideal orientations. Contours are in times-random units. 
each specimen and also show the COD values for some ideal orientations of special interest. The strongest single orientations present in specimens $A$ and $C$, processed using a single stage of cold reduction, were centered near ideal $\{111\}<112>$ orientations. Specimen B, processed with two stages of cold reduction, had a strong maximum near the ideal $\{110\}<001>$ orientation. Specimen D, also processed using two stages of cold reduction, had a weaker near $\{110\}<001>$ component than specimen $B$, but this component was significantly stronger for specimen $D$ than for either of the single stage specimens. As mentioned earlier, specimens $A$ and $B$ underwent significantly more grain growth during the final anneal than specimens $C$ and $D$ and the amount of grain growth may have a strong influence on the final textures.

To correlate magnetic properties with final textures, permeability and core loss values from Epstein samples cut at angles of $0^{\circ}, 20^{\circ}, 70^{\circ}$, and $90^{\circ}$ to the rolling direction for specimens $A$ and $B$ were fitted to expressions of the form of Equation (22). The appropriate values of $\phi_{4}(\alpha)$ and $\phi_{6}(\alpha)$ were calculated from the COD data, and values of the constants $A_{0}, A_{4}$, and $A_{6}$ were determined by least-squares. The form of Equation (22) was chosen over the equivalent form of Equation (8) to maintain consistency with the prior work of Hutchinson and Swift ${ }^{6}$ and conventional treatments of magnetic anisotropy.

The fourth-order texture parameter $\phi_{4}(\alpha)$ represents the weighted average of the direction cosine function $l^{2} \mathrm{~m}^{2}+$ $m^{2} n^{2}+n^{2} l^{2}$ for all crystallite orientations in a polycrystalline specimen while $\phi_{6}(\alpha)$ represents a weighted average of the direction cosine function $l^{2} \mathrm{~m}^{2} \mathrm{n}^{2}$. Least-squares analysis showed that the uncertainties in the sixth-order coefficients $A_{6}$ were larger than the calculated values of $A_{6}$ in all cases, so the sets of data were fitted to the fourth-order form of Equation (22):

$$
\bar{F}(\alpha)=A_{0}+A_{4} \phi_{4}(\alpha) \text {. }
$$

Hutchinson and Swift ${ }^{6}$ also found a fourth-order fit of the form of Equation (29) to be satisfactory to describe the variation of core loss with test direction in a low-silicon electrical steel.

Permeability and core loss data are plotted vs. $\phi_{4}(\alpha)$ in Figure 2 where the data for specimens $A$ and $B$ have been pooled. The permeability at $796 \mathrm{~A} / \mathrm{M}$ is known to be strongly dependent on the average orientation in electrical steels, 12 and the data for both specimens fit well to a common leastsquares line. This implies that any difference in permeability between samples of the two specimens cut in the same test direction is almost entirely due to texture differences. The pooled core loss data at $1.5 \mathrm{~T}$ are also described fairly well by a common least-squares line, which implies that texture differences are the most significant cause for core loss differences between the two specimens in any test direction. However, the data for two-stage specimen A appear to be slightly higher at any value of $\phi_{4}(\alpha)$ than those for singlestage specimen $B$. Other metallurgical factors such as grain 


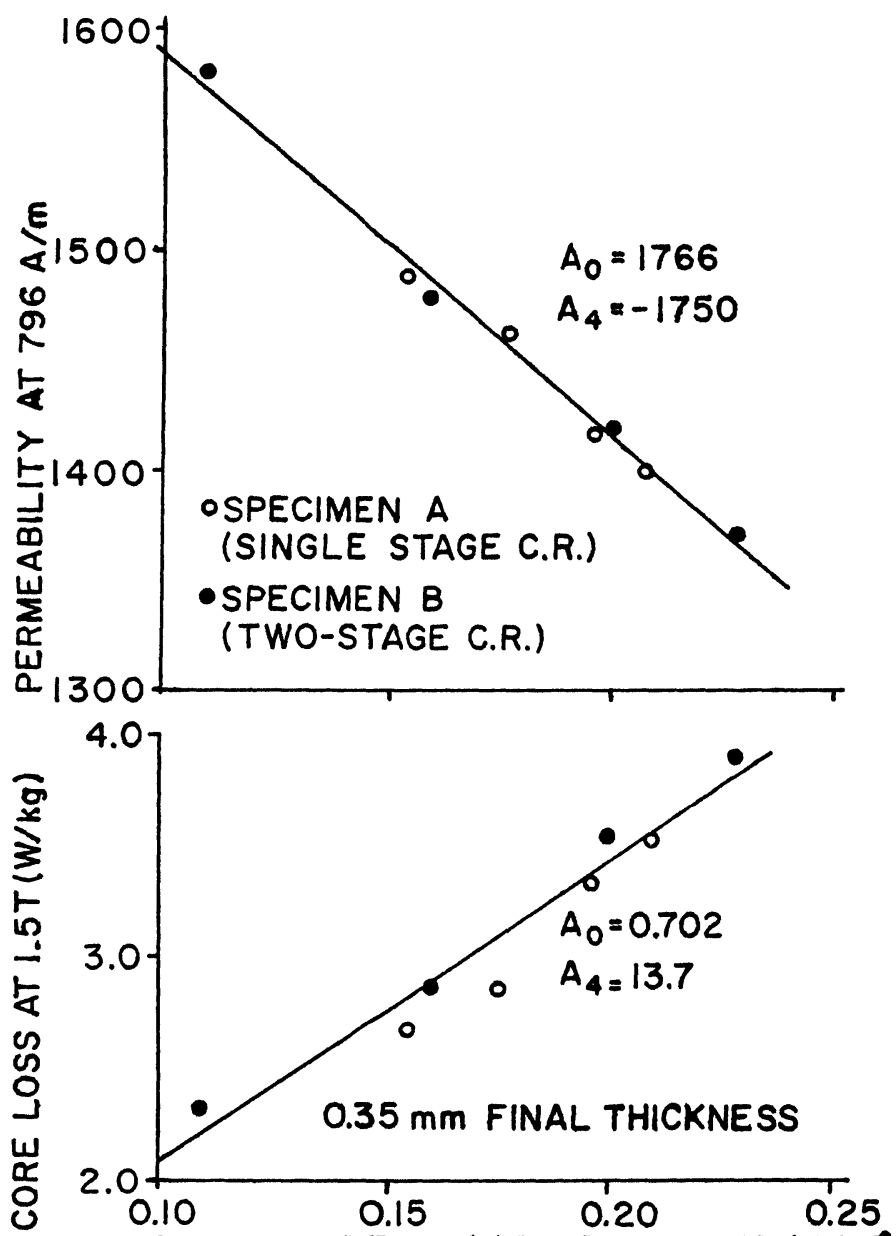

FOURTH ORDER TEXTURE PARAMETER $\varphi_{4}(\alpha)$

Figure 2. Permeability and core loss vs. fourth-order texture parameter.

size differences are known to affect core loss values, ${ }^{12}$ so there may be texture-independent factors which contribute to the apparent offset between the core loss values for the two specimens.

The fourth-order calculated values of permeability and core loss vs. test angle in the plane of the sheet for both specimens $A$ and $B$ are compared to the measured values in Figure 3. The agreement is generally good. The two-stage specimen $B$ has better values for both properties when tested parallel to the rolling direction, but has inferior properties at test angles near $50^{\circ}$. The much higher amount of material near the ideal $\{110\}<001>$ orientation for specimen $B$ compared to specimen A (see Figure l) is probably a major contributing factor to these differences.

Since the magnetic properties studied can be described with reasonable accuracy by equations which are linear with $\phi_{4}(\alpha)$, plots of the variation of $\phi_{4}(\alpha)$ vs. test angle can be 

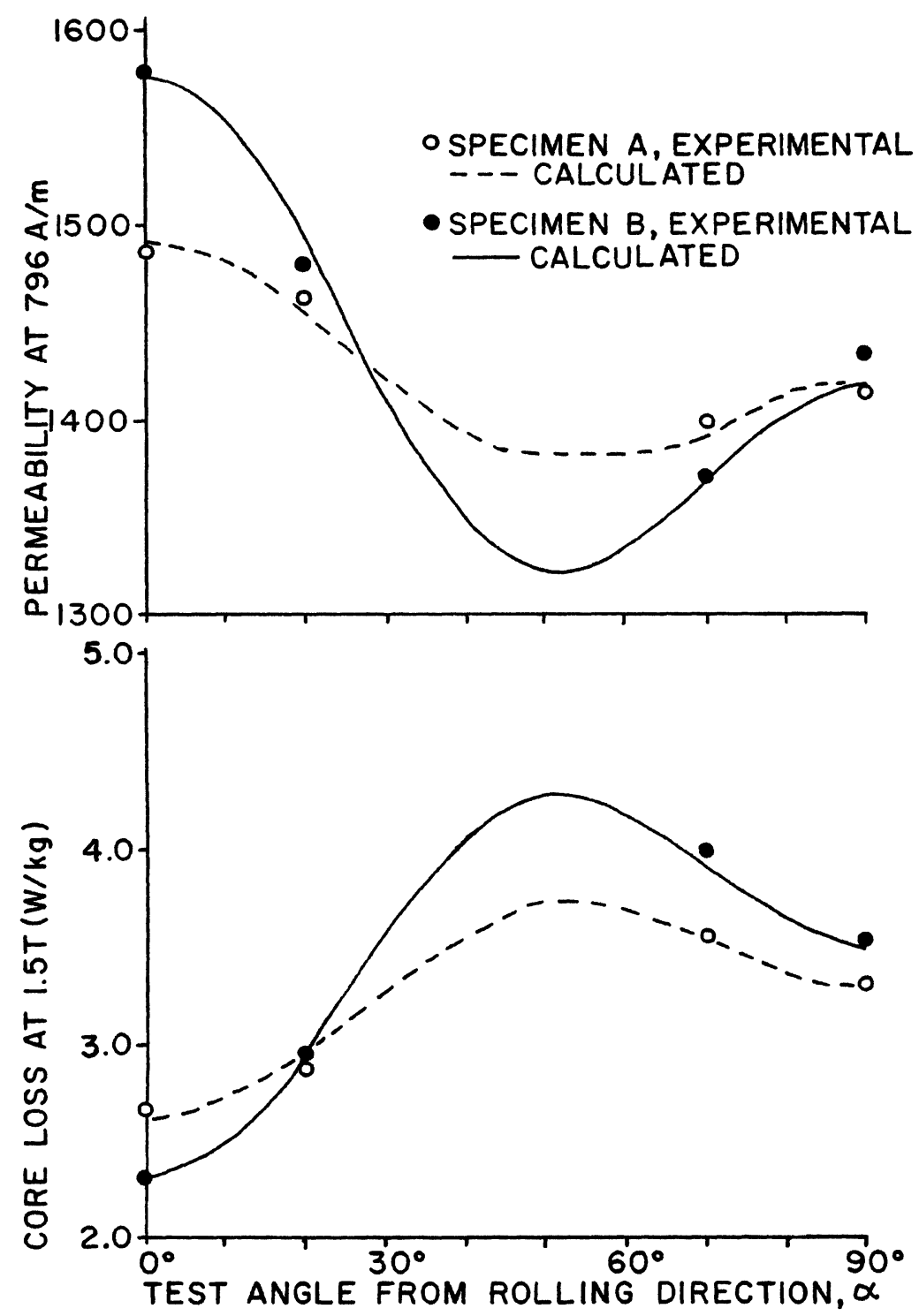

Figure 3. Calculated and measured variation of permeability and core loss in the plane of the sheet.

used to display the variation of permeability and core loss in the plane of the sheet if two or more test values are available. An example of such a plot is given in Figure 4 for specimen B. For materials with relatively weak multicomponent textures such as "nonoriented" electrical steels, such plots would seem to be of more immediate practical use for studying texture-property relationships than either conventional pole figures or COD sections.

It may also be noted that when a fourth-order expansion is adequate to describe magnetic property variations, a property value for a "random" sample (no preferred orientation) 


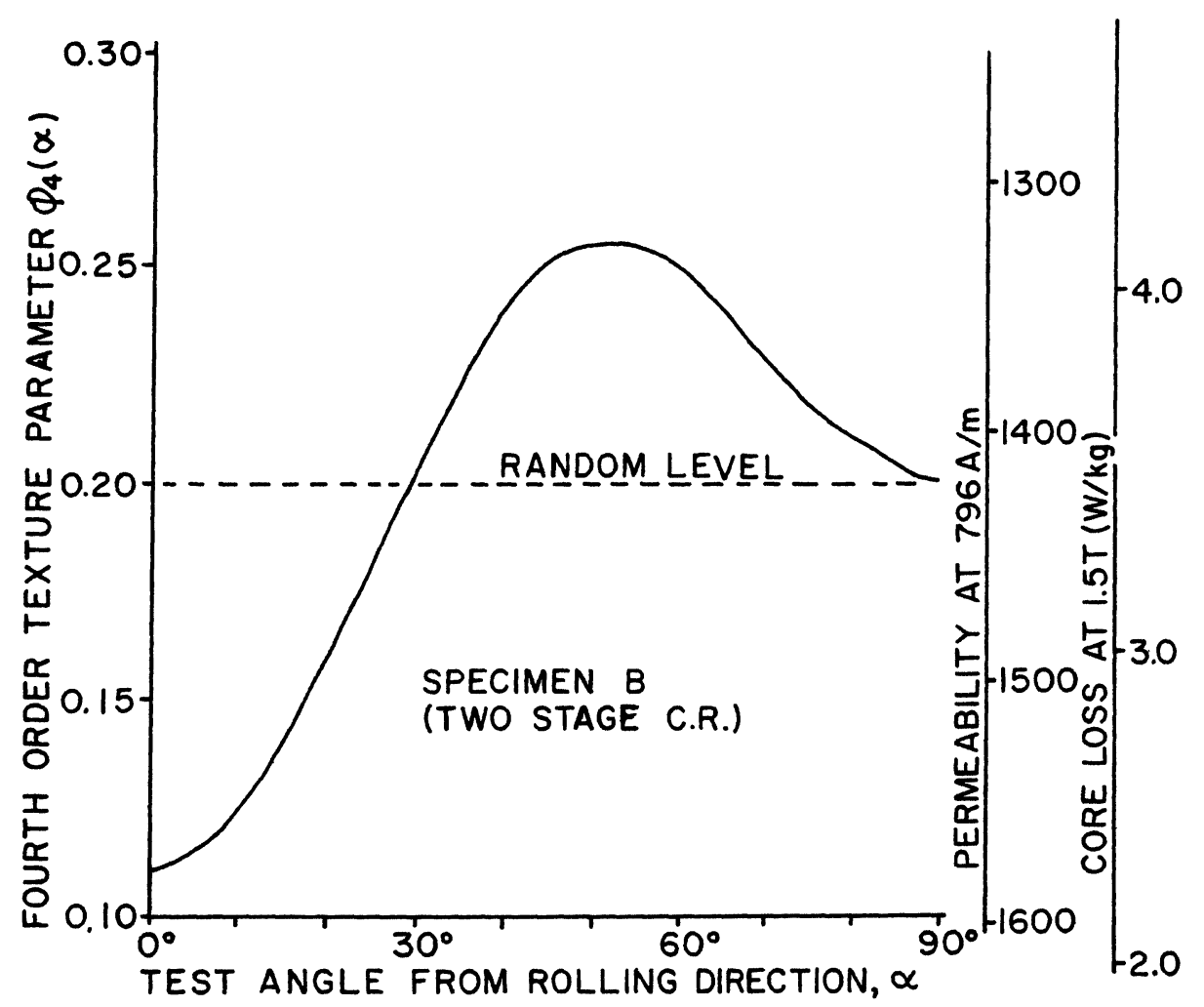

Figure 4. Variation of fourth-order texture parameter in the plane of the sheet and calculated fourth-order permeability and core loss.

may be obtained by setting $\mathrm{T}_{4}(\alpha)=0$ in Equation (8) or by setting $\phi_{4}(\alpha)=0.2$ in Equation $(29)$. The random property values of permeability and core loss for specimen $B$ are indicated in Figure 4. The random values are texture-independent properties for a given specimen and differences between the random values for two or more specimens represent property differences which are produced by factors other than texture. Hutchinson and Swift ${ }^{6}$ have pointed out that the constant term $A_{0}$ in Equation (29) represents the property value for a hypothetical specimen with the same microstructure but no misorientation. Differences among the $A_{0}$ terms for different specimens could also be used to measure texture-independent property differences.

The measured magnetic torque curves for single-stage specimen $C$ and two-stage specimen $D$ are given in Figure 5 along with torque values calculated from COD data using the fourth order form of Equation (28). A value of 380,000 ergs $/ \mathrm{cm}^{3}$ was used for the first anisotropy constant $\left(\mathrm{K}_{1}\right)$ both for calibrating the measured curves and calculating the fourth-order values. I Both specimens have low maximum torque values compared to highly-oriented silicon-iron specimens but two-stage specimen $D$ has a significantly higher maximum torque than single-stage sepcimen $C$. The agreement between measured and calculated torque values is generally good. 


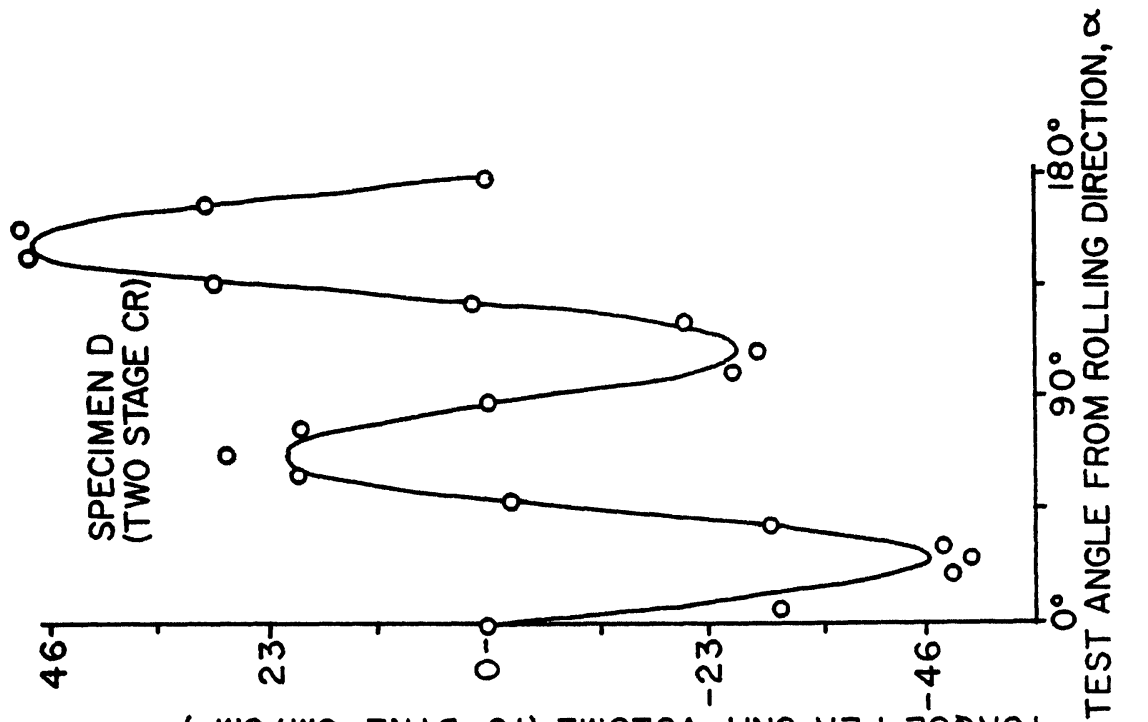

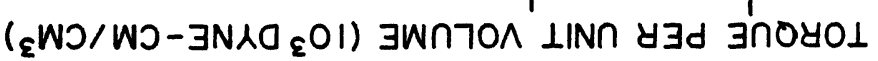

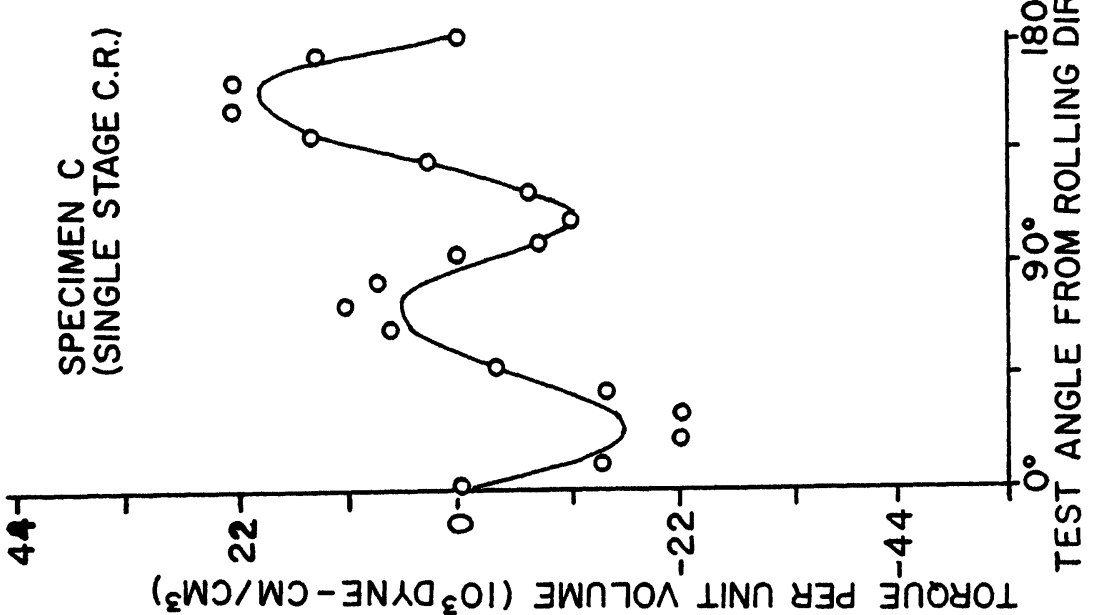


CONCLUSIONS

1) Expressions were derived for the dependence of magnetic properties on texture. The theoretical development generally parallels that of Bunge, but employs Roe's formalism.

2) Core loss, magnetic permeability and torque values for specimens of comparatively weakly-oriented electrical steels could be fitted to simple fourth-order equations.

3) The method, together with limited experimental data, enables one to express magnetic property variations in the plane of the sheet, to obtain property values for comparable specimens with no preferred orientation and to separate the magnetic properties into texture-dependent and textureindependent components.

\section{ACKNOWLEDGEMENTS}

We wish to thank D. R. Thornburg of Westinghouse Research Laboratories for obtaining the torque curves and D. W. Taylor, C. E. Ward, J. D. Evans, and G. S. Huppi of Research and Technology, Armco Inc., for helpful discussions.

\section{REFERENCES}

1. H. J. Bunge, Monatsberichte, 3,97 (1961).

2. H. J. Bunge, $Z$. Metallkde, 56,872 (1965).

3. R. J. Roe, J. Appl. Phys., $\frac{36}{36}, 2024$ (1965).

4. R. J. Roe, J. Appl. Phys., $\overline{37}, 2069$ (1966).

5. H. J. Bunge, Mathematische Methoden der Texturanalyse (AkademieVerlag, Berlin, 1969), p. 184.

6. W. B. Hutchinson and J. G. Swift, Texture, 1,117 (1973).

7. R. O. Williams, Trans. TMS-AIME, 242, 105 (1968).

8. J. Szpunar and M. Ojanen, Met. Trans. À, 6A, 561 (1975).

9. S. L. Lopata and E. B. Kula, Trans. AIME, 224, 865 (1962).

10. P. R. Morris and A. J. Heckler, in Advances in X-Ray Analysis (Plenum Press, New York, 1968), Vol. 11, p. 454.

11. W. M. Swift, W. T. Reynolds and D. R. Thornburg, IEEE Trans. on Mag., Vol. MAG-11, 1649 (1945).

12. M. F. Littmann, J. Appl. Phys., 38, 1104 (1967). 